\title{
A Comparative Study on Fear Expression Recognition Characteristics in First-episode Schizophrenic Patients
}

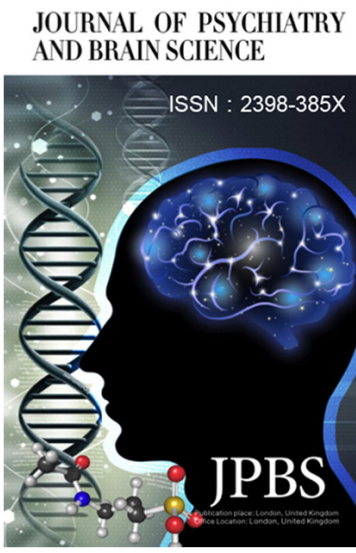

http://jpbs.qingres.com

\section{GOPEN ACCESS}

DOI: 10.20900/jpbs.20170012

Received: May 12, 2017

Accepted: July 18, 2017

Published: August 25, 2017

Copyright: ๑2017 Cain et al. This is an open access article distributed under the terms of the Creative Commons Attribution License, which permits unrestricted use, distribution, and reproduction in any medium, provided the original author and source are credited.
Ansi Qi ${ }^{1}$, Chengqing Yang ${ }^{2}$, Na Liu ${ }^{2}$, Junjuan $\mathrm{Zhu}^{2}$, Nan Huang ${ }^{2}$, Yi $\mathrm{Xu}^{2}$, Xiaofeng Guan ${ }^{1}$, Xiangyun Long ${ }^{1}$, Zheng Lu ${ }^{1,2^{*}}$

${ }^{1}$ Department of Psychiatry, Tongji Hospital of Tongji University, Shanghai 20065, P.R. China;

${ }^{2}$ Mental Health Center, School of Medicine, Shanghai Jiaotong University.

*Corresponding Author: Zheng Lu. Email: luzheng@tongji.edu.cn.

\section{ABSTRACT}

Objective: To explore the characteristics of fear expression recognition in the patients with first-episode schizophrenia.

Methods: Based on the case-control design, the Ekman facial expression database was used to research the subjects' overall response rate of low / high fear facial expression, accuracy rate of low / high level fear and its response time in the control group (28 cases) and the first-episode schizophrenic patient group (27 cases) with matching gender $(p=0.696)$, age $(p=0.255)$ and education level $(p=$ 0.895).

Results: The high level fear accuracy rate $(50.41 \pm 6.18)$ of case group was lower than that of the control group $(68.29 \pm 4.27, p=0.034)$. The response time $(362.84 \pm 25.23)$ of low-level fear of healthy control group was positively correlated with the accuracy rate $(21.79 \pm 2.28)$ $(r=0.494, p=0.008)$; the negative symptom of case group (23.35 \pm 10.56) was negatively correlated with the accuracy rate of high level fear $(r=-0.428, p=0.042)$.

Conclusion: The patients with first-episode schizophrenia have the disorder of high-level fear recognition and the accuracy rate of fear facial expression recognition can reveal the negative symptoms of the patients.

Keywords: First-episode Schizophrenia; Facial Expression Recognition; Response Time; Fear Expression

Schizophrenia is often accompanied by thinking, emotion, behavior, and cognitive disorders, and the schizophrenic symptoms can be divided into positive symptoms, negative symptoms, cognitive 
symptoms and emotional symptoms ${ }^{[1]}$. The emotional symptoms of schizophrenia patients can be expressed as the discordance between inner emotional experience and exterior environment. The treatment of facial expression by schizophrenia patients is different from that of the normal subjects [2]. Increasingly, Increasingly, schizophrenia patients' abilities were damaged in recognizing all kinds of facial expressions, but there exist differences among them ${ }^{[3]}$, and in all facial expressions, the incidence rate of fear-based negative emotion recognition disorders was significantly higher than that of positive emotions, like happiness ${ }^{[4]}$. Habel, et al. ${ }^{[5]}$ pointed out that this recognition difference existed in the American population. Vai, et al. ${ }^{[6]}$ also reported that the fear-based facial expression recognition defect also existed in the European population. In China's studies, Yang, et al. ${ }^{[7]}$ found that the schizophrenic inpatients had a low recognition rate for the aversion, but the difference of fear recognition rate was not significant. Hence, China has not yet clearly explored the characteristics of low fear recognition rate for schizophrenia, especially whether such is universal in the first-episode schizophrenia. In this study, the universality and relationship between first-episode schizophrenia and emotional treatment injury was demonstrated by comparing the recognition status of fear expression of first-episode schizophrenia patients.

\section{SUBJECTS AND METHODS}

\subsection{Subjects}

Thirty patients with schizophrenia and thirty healthy volunteers were collected in this study. To prevent the subjects from random answers, the subjects whose response rate was lower than $60 \%$ were considered to be uncooperative and removed (one case in health group and one case in study group). The subjects who failed to complete all tests were removed (one case in health group and two cases in study group). The composition of schizophrenic patients and healthy controls, including gender, age, and education level, was not statistically different, see Table 1.

Table 1. Basic Information of Healthy Control Group and Study Group

\begin{tabular}{llll}
\hline Variable & Control Group & Study Group & Kruskal Wallis Test \\
\hline Gender & $16 \mathrm{~F} / 12 \mathrm{M}$ & $14 \mathrm{~F} / 13 \mathrm{M}$ & 0.696 \\
Age & $26.79 \pm 1.142$ & $24.81 \pm 1.392$ & 0.255 \\
Education Level & $12.61 \pm 0.389$ & $12.85 \pm 0.458$ & 0.895 \\
\hline
\end{tabular}

The patients of first-episode schizophrenia group were all from the outpatient department of Shanghai Mental Health Center from December 2015 to December 2016. They complied with the diagnostic standards of schizophrenia in Diagnostic and Statistical Manual of Mental Disorders (DSMIV). The disease course of the patients who did not take medications was shorter than two years, and the patients' IQ was more than 70 assessed on the Wechsler Intelligence Scale. The patients or subjects had no mental retardation, nor neurological disease, nor major physical illness; they had no history of alcohol or drug abuse and dependence. They did not receive the $\mathrm{MECT}$, and did not have long-term use of anticholinergic agents, benzodiazepines, and other drugs that may affect the cognitive function.
The volunteers of healthy control groups were all recruited from community volunteers and hospital staff in China, and matched with the patients. Clinical interview for DSM-IV-TR axis T disorder (SCID rating scale) was used to exclude those who have a history of schizophrenia and positive mental disorders. Volunteers' IQ was more than 70, assessed on the Wechsler Intelligence Scale; they had no history of brain trauma, alcohol, or drug abuse and dependence.

This study was approved by the Ethics Committee of Tongji Hospital of Tongji University and the written informed consents were all obtained from the participants. 


\subsection{Research Methods}

\subsubsection{General Data Collection and Scale Assessment}

The demographic data was collected and surveyed from the subjects of the two groups, including gender, age and education level. The patients were clinically assessed, including the use of PANSS to assess their psychiatric symptoms. The scale assessment performers were two senior doctors from Shanghai Mental Health Center. The Kappa value of the consistency test was 0.87 .

\subsubsection{Experimental Materials: software E-prime 2.0 was used}

The faces used in the experiment were selected from the Ekman standard black-white face database. The facial expression pictures were composed of two male faces and two female faces. The "fear" faces were divided into ten levels according to the extent of performance from $0 \%$ to $100 \%$.

\subsubsection{Experimental Contents: the experiments were designed into blocks}

Each block contains 132 trials and each trial was composed of fixation points ("+") and expression pictures. Each picture exposed for $400 \mathrm{~ms}$, followed by fixation points, with an exposure time ranging from $800 \mathrm{~ms}$ to $1600 \mathrm{~ms}$. The subjects were required to recognize the expression at this moment by pressing the keys. The patients rested for $10 \mathrm{~s}$ at the end of each block, followed by the next experiment. Two blocks were required for each expression recognition experiment, and an experiment lasted about 20 minutes. Each subject was required to receive the pre-test training for three minutes before taking an experiment.

\subsubsection{Statistical Analysis: the data were statistically analyzed using software SPSS20.0. An alpha of 0.05 is used as the cutoff for significance}

The "fear" expression was divided into two levels: low-level fear ranged from 0 to $50 \%$ and high-level fear ranged from $51 \%$ to $100 \%$. The rank sum test was performed on the accuracy rate of low/highlevel fears in the case group and the control group. The bivariate correlative analysis was performed on age, gender, group type, education level, low-level fear response accuracy rate, low-level fear response time, high-level fear response accuracy rate, highlevel fear response time, PANSS positive symptom score, negative symptom score, general symptom score, total score, and other variables.

\section{RESULTS}

The recognition accuracy rate of fear expression at different degrees in the two groups was not distributed normally. From the $U$ test, the recognition accuracy rate of high-level fear expression in firstepisode schizophrenia case group was lower than that of the control group, and the difference was statistically significant $(p=0.034)$, see Table 2 . and Fig. 1. The recognition accuracy rate of low-level fear in the two groups was not statistically significant $(p<0.05)$. The response time of different degrees of fears in the two groups was not statistically significant $(p>0.05)$.

Table 2. Comparison of Accuracy Rate and Response Time for Fear Expression between Control Group and Case Group

\begin{tabular}{|c|c|c|c|c|}
\hline \multirow{2}{*}{ Group } & \multicolumn{2}{|c|}{ Low Level Fear } & \multicolumn{2}{|c|}{ High Level Fear } \\
\hline & $\begin{array}{c}\text { Accuracy Rate } \\
(\%)\end{array}$ & $\begin{array}{c}\text { Mean Response } \\
\text { Time (ms) }\end{array}$ & $\begin{array}{c}\text { Accuracy Rate } \\
(\%)\end{array}$ & $\begin{array}{c}\text { Mean Response } \\
\text { Time (ms) }\end{array}$ \\
\hline $\begin{array}{l}\text { Schizophrenia } \\
\text { Group }(n=27)\end{array}$ & $16.07 \pm 2.28$ & $392.69 \pm 20.83$ & $50.41 \pm 6.18$ & $414.18 \pm 25.91$ \\
\hline $\begin{array}{l}\text { Control Group } \\
(n=28)\end{array}$ & $21.79 \pm 2.28$ & $362.84 \pm 25.23$ & $68.29 \pm 4.27$ & $374.08 \pm 29.46$ \\
\hline $\mathrm{u}$ test & 0.083 & 0.297 & $0.034^{*}$ & 0.502 \\
\hline
\end{tabular}




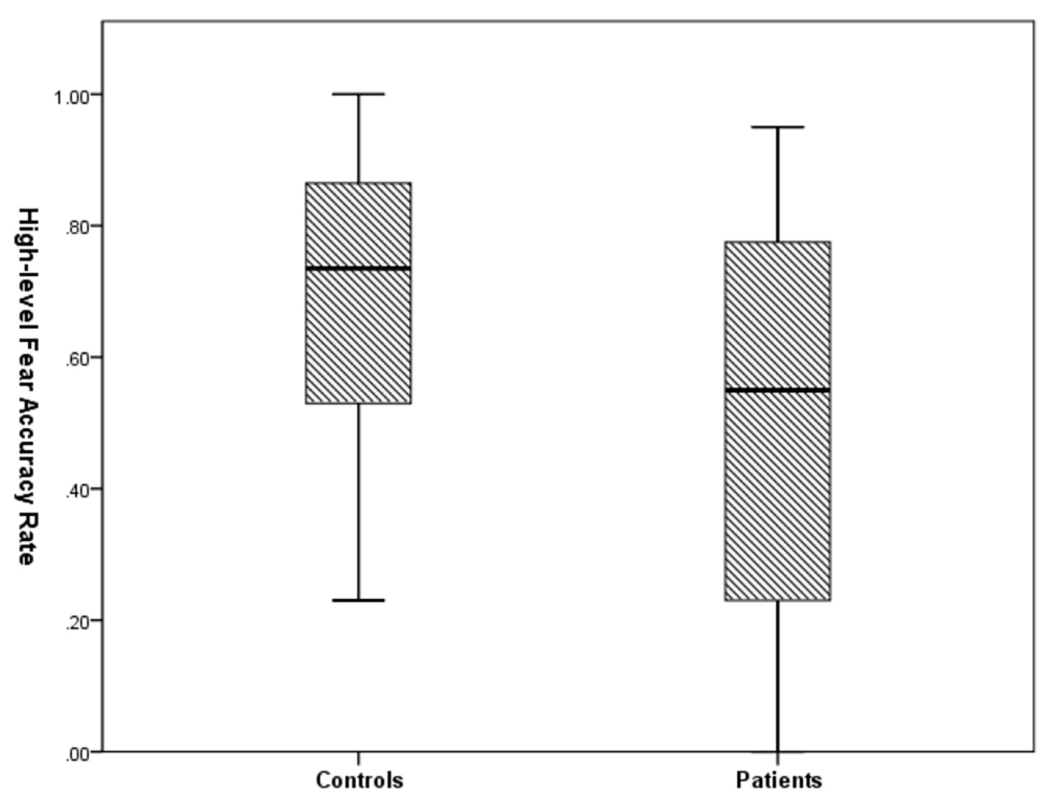

Fig.1 Comparison of High-level Fear Recognition Accuracy Rate between Control Group and Case Group

The age, gender and education level of patients in the case group were not significantly correlated with the expression response time and accuracy rate. The response time of low level fear of the volunteers in the control group was positively correlated with the accuracy rate $(r=0.494, p=0.008)$. The correlative analysis between the accuracy rate and response time and the clinical symptom of the case group was further performed. The positive symptom score
$(24.74 \pm 8.07)$, general symptom score $(48 \pm 11.82)$ and total score $(96.18 \pm 26.85)$ of the case group obtained based on the PANSS scale rating were not significantly correlated with the response time and accuracy rate for the expressions of different degrees. The negative symptom score $(23.35 \pm$ 10.56) was negatively correlated with the accuracy rate of high-level fear expressions $(r=-0.428, p=$ 0.042), see Fig. 2.
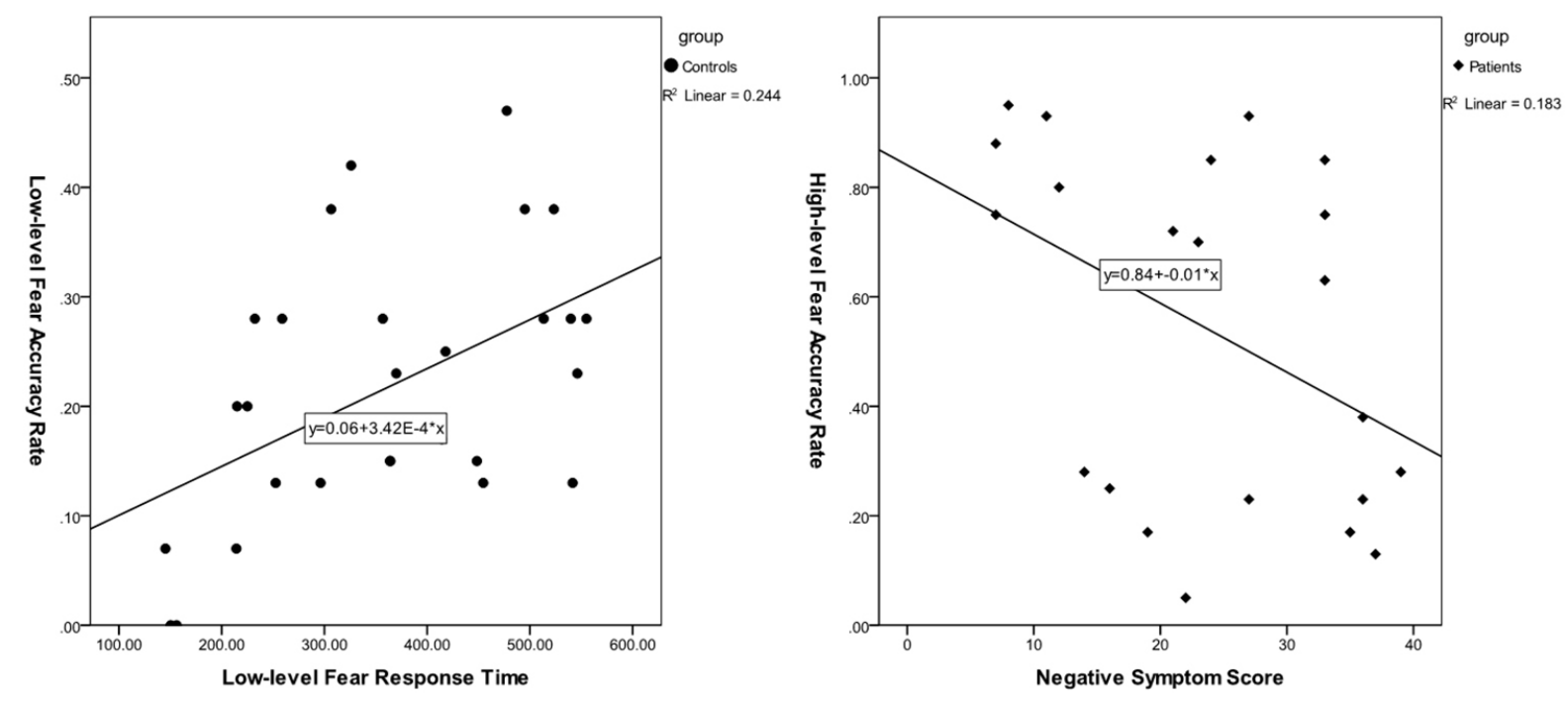

Fig.2 Correlation between Low-level Fear Response Time and Accuracy Rate in Control Group, and between Negative Symptom Score and High-level Fear Accuracy Rate in Case Group 


\section{DISCUSSION}

A significant recognition disorder for fear expressions was found in the patients with first-episode schizophrenia in this study. The high-level fear is relatively easier to be recognized than the low-level fear, but the patients' accuracy rate of recognizing the high-level fear is significantly lower than that of the control group. The negative symptom of the schizophrenic patients is negatively correlated with the recognition accuracy rate of high-level fear expressions.

The basic facial expressions include pleasure, disgust, fear, anger, sadness, etc. ${ }^{[8]}$ The schizophrenic patients' ability to recognize all expressions is damaged ${ }^{[9]}$, but their recognition ability of the fear expressions is significantly worse than other emotions, The recognition ability of fear face was corrected with the positive / negative symptoms, emotional symptom, and recognition symptoms ${ }^{[6]}$. It is presumed that the performance of both patients and controls is not ideal due to difficulty in recognizing the low-level fear expressions, whereas it is not as difficult to recognize the high-level fear expressions. The low accuracy rate of patient group is more indicative of the facial treatment defects of schizophrenia population of fear expressions. In this study, we observed that the higher score of the patient's negative symptoms reached, there would be the greater indifference of the patients, the lower recognition rate of high-level fear expressions, and the more serious the damage of recognition ability of facial expressions. Some studies show that a low recognition rate of schizophrenic patients for the fear expressions was due to the visual impairment ${ }^{[10]}$. EEG studies have further shown that the amplitude of patients' occipital lobes P100, N170 and N250 when performing the recognition task of fear facial expressions was less than that of the control group and the latency was shorter than that of the control group ${ }^{[11]}$. This indicates that the patients shy away from the fear emotion, with a shorter eye contact time when facing a fear face. The schizophrenic patients have more negative emotional experiences ${ }^{[12]}$, but a significantly insufficient recognition of fear expression was found in the schizophrenic patients in this study. In combination with the previous studies, we hypothesize that the patients with first-episode schizophrenia have more eye avoidance in the face of fear expressions, thus resulting in the dysfunction of their facial expression processing system and prone to the false recognition. The amygdalebased limbic system is mainly associated with the fear processing and threat detection. Increasingly, evidence shows that the incomplete activation of amygdale and the disruption of emotional network function in the schizophrenic patients has impaired the recognition processing of their facial expressions [13-14]. These studies have further confirmed our conclusion. Moreover, the disruption of brain network function requires the schizophrenic patients to mobilize more brain regions to identify the facial expressions ${ }^{[15]}$, therefore, the response time needed is longer than that of the controls. In this study, the average response time of the patients for the lowlevel or high-level disgust expressions is longer than that of the controls, but the results were not statistically significant. Presumably, this is due to the small sample size.

It was also found in this study that the accuracy rate of the control group increased with the increase of response time to the low-level fear, while there was no similar correlation in the high level fear. Considering that the recognition level of low-level fear is significantly lower than that of the high-level fear, the healthy population needs to increase the response time in order to improve the recognition rate when recognizing the difficult fear expressions. No similar finding was obtained in the study group, which indicates that the patients' recognition ability of low / high-level fear expressions cannot be improved with the prolonging of the response time. Therefore, the patients have defects in recognizing these two levels of fear expressions, and this recognition defect cannot be compensated by prolonging the response time. Moreover, the patients are more likely to recognize the fear expression as other emotions. This further suggests an inherent mechanism defect of recognizing the fear expressions in the patients with first-episode schizophrenia. Furthermore, the correlation analysis shows that the patients' recognition accuracy rate of high-level fear expressions and negative symptoms were negatively correlated, but this correlation does not exist in the scores of positive symptoms and general symptoms. It indicates that the patients with a worse recognition ability of facial expressions may experience more significant emotional indifference, while the emotions of the positive symptom-based patients may not be necessarily impaired.This is consistent with clinical experiences, and additionally, it is also indicates that the positive symptom and negative symptom may be based on different brain functional areas and that they have different brain functional network connections. This is consistent with the current studies ${ }^{[6]}$.

In conclusion, the behavioral analysis of the recognition task of facial fear expressions shows that the patients with first-episode schizophrenia have a disorder of faulty fearful emotion recognition, most evident in the obvious fearful expressions. This 
recognition defect cannot be compensated by relying on the increase of response time, and the accuracy rate of fear facial expression recognition can reveal the negative symptoms of the patients. The small sample size is the main defect of this study. The further studies shall need to expand the sample size, increase the follow-up frequency of the patients, and further explore the recognition disorder of fear facial expressions in first-episode schizophrenic patients with respect to anatomy and functional structure, in combination with the magnetic resonance, brain evoked potential, and other means, so as to provide the reference basis for the clinical treatment of the first-episode schizophrenia.

\section{FUNDING SUPPORT}

This project is supported by the National Key Research and Development Program of China (No.: 2016YFC1306805), the National Natural Science Foundation of China (No.: 81471359) and The Chinese-Western Medicine Guiding Project of Shanghai Science and Technology Commission (No.:14411963400).

\section{REFERENCES}

1. Nielsen RE, Levander S, Kjaersdam Telleus G, Jensen SO, Østergaard Christensen T, Leucht S. Second-generation antipsychotic effect on cognition in patients with schizophrenia--a meta-analysis of randomized clinical trials. Acta Psychiatr Scand. 2015; 131(3): 185-196.

2. Leppanen JM, Niehaus DH, Koen L, Du Toit E, Schoeman R, Emsley R. Emotional face processing deficit in schizophrenia: A replication study in a South African Xhosa population. Schizophr Res. 2006; 84(2-3): 323-330.

3. Mothersill O, Morris DW, Kelly S, Rose EJ, Bokde A, Reilly R, Gill M, Corvin AP, Donohoe G. Altered medial prefrontal activity during dynamic face processing in schizophrenia spectrum patients. Schizophr Res. 2014; 157(1-3): 225230.

4. Leitman DI, Loughead J, Wolf DH, Ruparel K, Kohler CG, Elliott MA, Bilker WB, Gur RE, Gur RC. Abnormal superior temporal connectivity during fear perception in schizophrenia. Schizophr Bull. 2008; 34(4): 673-678.

5. Habel U, Gur RC, Mandal MK, Salloum JB, Gur RE, Schneider F. Emotional processing in schizophrenia across cultures: standardized measures of discrimination and experience.
Schizophr Res. 2000; 42(1): 57-66.

6. Vai B, Papa GS, Poletti S, Radaelli D, Donnici E, Bollettini I, Falini A, Cavallaro R, Smeraldi $E$, Benedetti F. Abnormal cortico-limbic connectivity during emotional processing correlates with symptom severity in schizophrenia. Eur Psychiat. 2015; 30(5): 590597.

7. Yang CQ, Lu Z, Wang JJ, Chen FZ, Liu N, He LY, Huang N, Li H, Tang YY. Research in Facial Recognition Examined by Eventrelated Potentials in Patients with First-episode Schizophrenia. Chin J Psychiat. 2012; 45(2): 7479.

8. Fakra E, Salgado-Pineda P, Delaveau P, Hariri $A R$, Blin O. Neural bases of different cognitive strategies for facial affect processing in schizophrenia. Schizophr Res. 2008; 100(1): 191-205.

9. Yang $\mathrm{C}$, Zhang $\mathrm{T}$, Li Z, Heeramun-Aubeeluck A, Liu N, Huang N, Zhang J, He L, Li H, Tang Y, Chen F, Liu F, Wang J, Lu Z. The relationship between facial emotion recognition and executive functions in first-episode patients with schizophreniaand their siblings. BMC Psychiat. 2015; 15: 241.

10. Adolphs R. What does the amygdala contribute to social cognition? Ann N Y Acad Sci. 2010; 1191: 42-61.

11. Yang C, Zhang T, Li Z, Heeramun-Aubeeluck A, Liu N, Huang N, Zhang J, He L, Li H, Tang Y, Chen F, Wang J, Lu Z. Changes in eventrelated potentials in patients with first-episode schizophrenia and their siblings. BMC psychiat. 2017; 17(1): 20.

12. Morris RW, Sparks A, Mitchell PB, Weickert CS, Green MJ. Lack of cortico-limbic coupling in bipolar disorder and schizophrenia during emotion regulation. Transl Psychiatr. 2012; 2: e90.

13. Doll A, Holzel BK, Bratec SM, Boucard CC, Xie $X$, Wohlschläger AM, Sorg C. Mindful attention to breath regulates emotions via increased amygdala-prefrontal cortex connectivity. Neurolmage. 2016; 134: 305-313.

14. Bjorkquist OA, Olsen EK, Nelson BD, Herbener ES. Altered amygdala-prefrontal connectivity during emotion perception in schizophrenia. Schizophr Res. 2016; 175(1-3): 35-41.

15. Fakra E, Salgado-Pineda P, Delaveau P, Hariri $A R$, Blin O. Neural bases of different cognitive strategies for facial affect processing in schizophrenia. Schizophr Res. 2008; 100(1): 191-205. 